\title{
ANALYSIS OF THE PERTURBING ACTIONS ON THE CONSTANT SPEED DRIVE INPUT LINK
}

\author{
MIKHAYLOV, M[axim]; STAZHKOV, S[ergey] \& CVETKOV, V[ladimir]
}

\begin{abstract}
Constant speed drive are stabilizing systems, and in such systems the reference signal is constant, and the main operating mode of these systems is aimed at eliminating or reducing errors caused by disturbances. The analysis of disturbances on CSD has practical value for a creation of a drive with the required precision characteristics, allowing studying the interconnection of the impact distribution and static characteristics of the drive and installation power of its hydraulic machines.
\end{abstract}

Keywords: mechatronics, hydraulics, power drives, control systems

\section{INTRODUCTION}

Constant speed drive (CSD) are stabilizing systems, and in such systems the reference signal is constant, and the main operating mode of these systems is aimed at eliminating or reducing errors caused by disturbances.

Constant speed drives input link, mounted on selfpropelled machines, is directly connected to the shaft of the main engine, as in the conventional PTO system with independent drive. CSD output link is connected with the load, which value can vary from zero to a maximum. Therefore, the major perturbing impact on the work of CSD, determining the conditions of its operation, will be two factors: the operating conditions of the groundpropelled machines main engine and loading variations on the CSD output link [5].

\section{CSD DESCRIPTION}

The proposed CSD is based on the hydromechanical transmission. Such system is designed for stepless transformation of variable speed of the input shaft into constant speed of the output shaft and its maintenance. The CSD can be directly connected with the electric generator output shaft that will ensure its constant speed and correspondingly constant value of produced voltage.

The CSD power unit consists of input shaft, threelink compensation gear and output shaft, which are connected through power transmissions. The variable moment is transformed during the operation into constant moment by the compensation gear. This moment in turn comes to the generator shaft. Due to its kinematic scheme the compensation gear has variable transmission ratio. Variable transmission ratio has to change depending on input moment value to maintain constant moment on output. The executable hydraulic circuit is designed for working off the necessary transmission ratio of compensation gear and for reinforcement of transitional moment. The hydraulic circuit includes unregulated hydraulic motor that is kinematically connected with one of the compensation gear links, and regulated hydraulic pump that is hydraulically connected with the first generator. The use of hydraulic drive significantly increases the drive specific output compared to other types of drives of similar dimensions while maintaining a high level of coefficient of efficiency [4].

The block sheme of CSD shown in Fig. 1. In his sheme: variable displacement hydraulic motor VDM, fixed displacement hydraulic motor FDM, three-link differential $D$, actuating motor $A M$,consumer motor (e.g., a direct-current generator) $C M$, velocity sensors $V S 1$ and $V S 2$, main controller $M$,amplifier $A S$, electrohydraulic converter $E H C$.

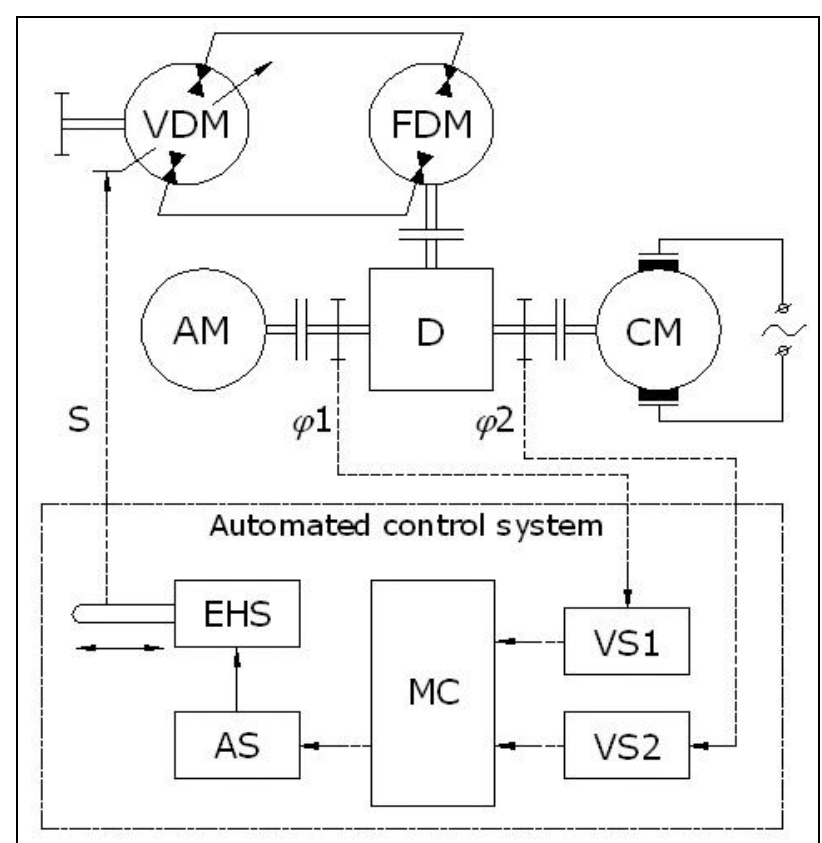

Fig. 1. Block sheme of CSD

The drive is equipped with speed sensors of input and output shafts, turning angle of controlled pump cylinder unit and pressure sensors of hydraulic fluid in hydraulic circuit and hydraulic generators. The feedback signal of these sensors comes into the control.

Primary analysis of main characteristics of the generation system based on CSD with traditional system based on power electric transducers showed that the offered solution has a better characteristic of specific output while other basic parameters being equal. In fact it 
means that having similar outputs of the energy generation systems, the system based on CSD will have significantly lower indicators of weight and dimensions, that will lead to the weight decrease of the generation system body (and consequently to the lightening and reduction in cost of transportation and installation). The main results of the analysis are presented in Tab. 1.

\begin{tabular}{|c|c|c|}
\hline Parametr & CSD & Power electric \\
\hline Efficiency & $92-95 \%$ & $95 \%$ \\
\hline Accuracy & $4 \%$ & $3-4 \%$ \\
\hline Specific power & $1,6-1,8 \mathrm{~kg} / \mathrm{kW}$ & $4 \mathrm{~kg} / \mathrm{kW}$ \\
\hline Relative olume & $0,0029 \mathrm{~m}^{3} / \mathrm{kW}$ & $0,0072 \mathrm{~m}^{3} / \mathrm{kW}$ \\
\hline
\end{tabular}

Tab.1. Main results of the characteristics analysis

\section{SCIENTIFIC PROBLEM}

Operation of volume hydromechanical transmission (VHT) in the CSD mode has a series of important features. In particular, from a perspective of the volume hydromechanical drive it is the unusual operation of hydraulic generators within the drive: angular speed of the motor changes to zero and a hydraulic pump parameter takes zero values, i.e. in the process of changing the gear ratio the operation modes of hydraulic generators are changing while the operation modes of input and output links of transmission are constant. This process has a negative impact on the value of the HCSD output shaft positioning accuracy, which is one of the determinants of performance quality of the drive. The above aspects of the drive operation are nowadays the subject of scientific researches.

The main objective of the study of CSD modes is to identify the requirements for obtaining a given accuracy to maintain a stable rate, while ensuring the minimum weight and dimensions at maximum coefficient of efficiency of the drive and to work out the requirements for the control system on the basis of received data.

In the process it became clear that during a regulation of the pump parameter propagation the unregulated motor passes through the area of zero turns, changing its direction of rotation. As noted above, this is a nonspecific mode of operation and it creates a main difficulty in designing drives of this type. It is explained by the fact that at the boundaries of the zone of zero velocity the hydraulic motor stops, as the whole volume of feeding is to compensate the leak of the fluid (the value of volume loss increases sharply). Within this zone the generator is not able to compensate for leakages. That's why along with the hydraulic motor speed the pressure in the hydraulic drive is gradually reducing to zero. The speed of drive output shaft decreases in the period when hydraulic generator passes through the zero zone. This leads to a failure of the drive output shaft rotation rate and consequently to the failure of generator rotation speed. It is important that the failure of the generator speed should not exceed $4 \%$, as this accuracy determines the quality of produced AC current, especially its frequency stability.

To compensate for this factor, it was proposed that decision. CSD kinematic sheme must provide work in the areas of transfer relations, where the probability of getting into zone of zero velocity is minimal.

For this purpose, the work of the drive was investigated the influence of external disturbances occurring on the output link, and made their quantitative and qualitative analysis.

\section{DISTURBANCES ANALYSIS}

As the power plant, that is the main engine for ground mobile vehicles, reciprocating internal combustion engines are typically used. On these machines the most widely used are diesel engines. Capacity of modern diesel engines used in mobile land vehicles, is up to 1000 $\mathrm{kW}$. Power takeoff for CSD today, is now is not higher than $35 \ldots 60 \mathrm{~kW}$. We can make an assumption that the capacity of the drive motor is infinitely large compared to the capacity taken by CSD for a generator drive.

Input parameters are dependent on many factors previously unknown (road category, driving mode, etc.) and can be determined by statistical methods. Input action on a drive is characterized by the spectral density distribution determined from experimental data, with a value depending on the form of the approximating.

For the probabilistic description of the engine speed deviation from the nominal the correlation function is used (1).

$$
K \bar{\omega}_{\text {д }}(\tau)=\lim _{T \rightarrow \infty} \frac{1}{T} \int_{0}^{T} \bar{\omega}_{\text {д }}(t) \cdot \bar{\omega}_{\text {д }}(t+\tau) d t(1)
$$

The correlation function is defined by processing the statistical results of measure the speed of rotation of the PTO shaft when the vehicle moves under various conditions by a known procedure.

The transition to the spectral density of the input action is done via the cosine transform (2).

$$
S \bar{\omega}_{\text {д }}(\omega)=\frac{2}{\pi} \int_{0}^{\infty} K \bar{\omega}_{\text {д }}(\tau) \cdot \cos (\omega \tau) d \tau
$$

In equation (2): $\omega$ - alternating frequency of the input action, in this case, the speed of rotation of the engine room.

The results of measurements speed PTO shaft when the vehicle is on the ground are used as initial data for the determination of the spectral density function of the input action. The graph of the shaft speed of heavy vehicle during acceleration for 140 seconds on the interurban highway is known[1]. It is stated that the non-stationary engine operating mode when driving that highway is about $50 \%$ of the time. Thereby it is possible to finish the graph of the vehicle shaft speed variations (Fig. 2).

Based on experimental data the autocorrelation function of the input action can be obtained, 
characterizing the deviation of engine speed from the nominal. Fig. 3 is a chart analysis of the autocorrelation function.

Chart analysis of the autocorrelation function, which was built for the given distribution, shows that there is a decreasing trend of the correlation coefficient from the maximum value to 0 when frequency is decreasing. Thus, we can conclude that the function decays asymptotically approaching 0 , which indicates that the ergodicity of the process (i.e., a random function as time of its observation increases $\mathrm{t} \rightarrow \infty$, the average value matches the mathematical expectation over the set).

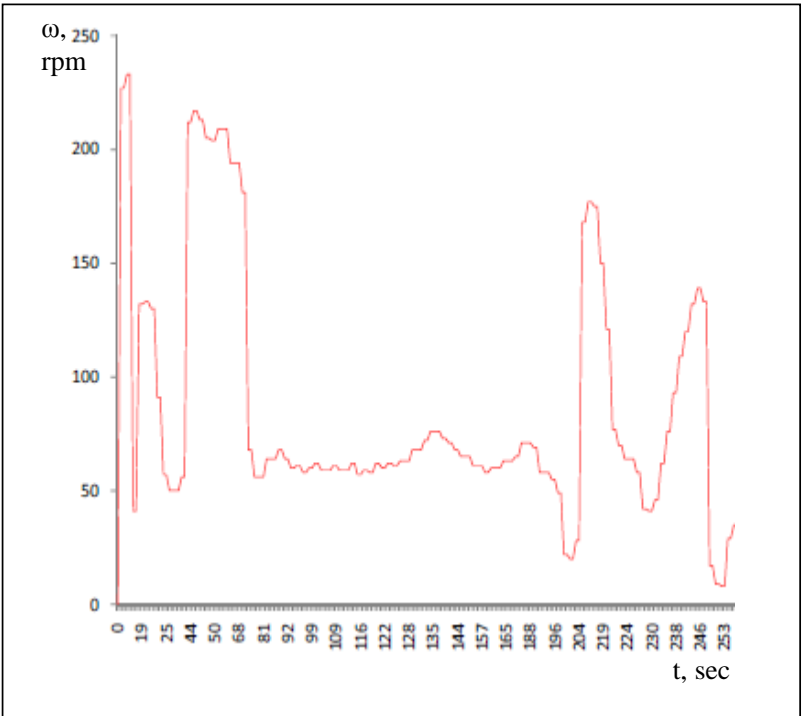

Fig. 2. Graph of the vehicle shaft speed variations

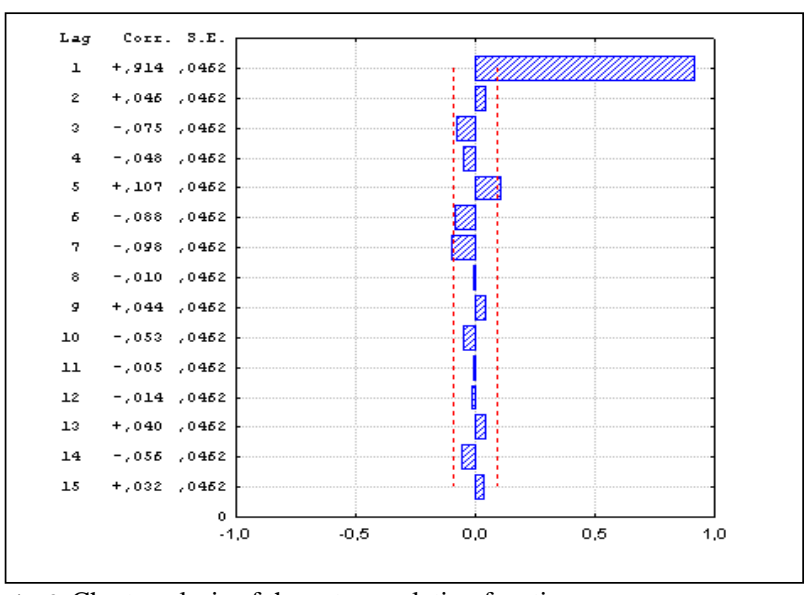

Fig. 3. Chart analysis of the autocorrelation function

For obtaining the spectral density the input action the autocorrelation function should be subjected to a cosine Fourier transform. In what follows points forming a graph of the spectral density distribution the input action on the frequency of variations approximated by expressions that may further be suitable for later use in CSD control system optimization. Such expression will be the quotient of two even polynomials [2] (3).

$$
S \bar{\omega}_{\text {д }}(\omega)=\frac{a_{p}\left(\omega^{2}\right)^{p}+a_{p-1}\left(\omega^{2}\right)^{p-1}+\ldots+a_{0}}{b_{q}\left(\omega^{2}\right)^{q}+b_{q-1}\left(\omega^{2}\right)^{q-1}+\ldots+b_{0}}
$$

In equation (3): $a_{i}, i=0 \ldots p \quad$ и $b_{j}, j=0 \ldots q-$ constant coefficients.
Approximation of the expression represented in the form generally is a nonlinear making it difficult finding the constant coefficients. Therefore proposed a special calculation method consisting in that the coefficients of the denominator are given some initial values, and then the denominator is multiplied by value of the spectral density, obtained by numerical integration [2]. Incidentally, the problem is reduced to the usual polynomial approximation. In addition, further analysis of the data on which the graphs of the spectral density functions were made, it is possible to obtain specific distribution of the values the input action. Fig. 4 shows a chart of spectral density function without approximation, Fig. 5 - chart with approximation.

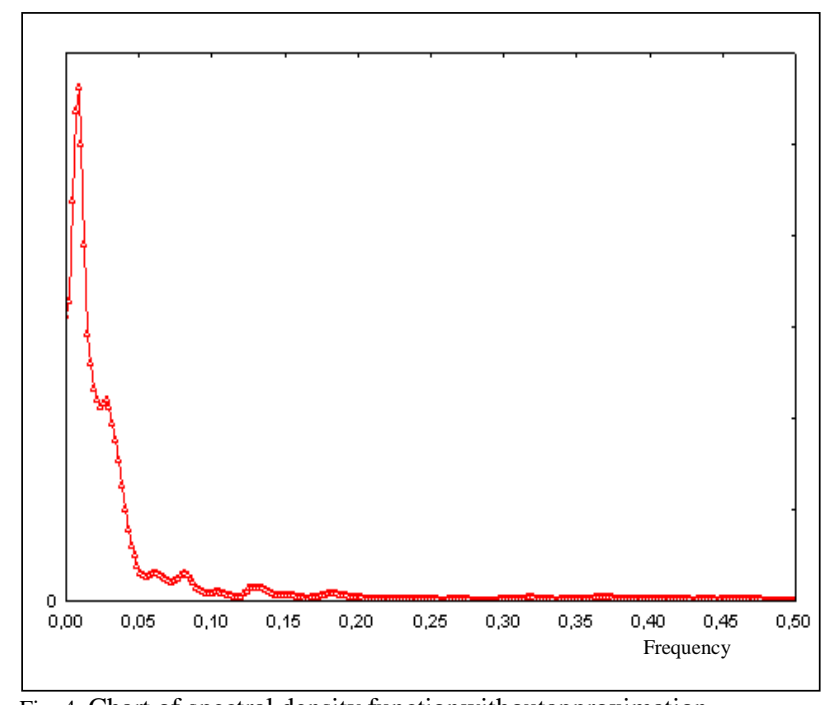

Fig. 4. Chart of spectral density functionwithoutapproximation

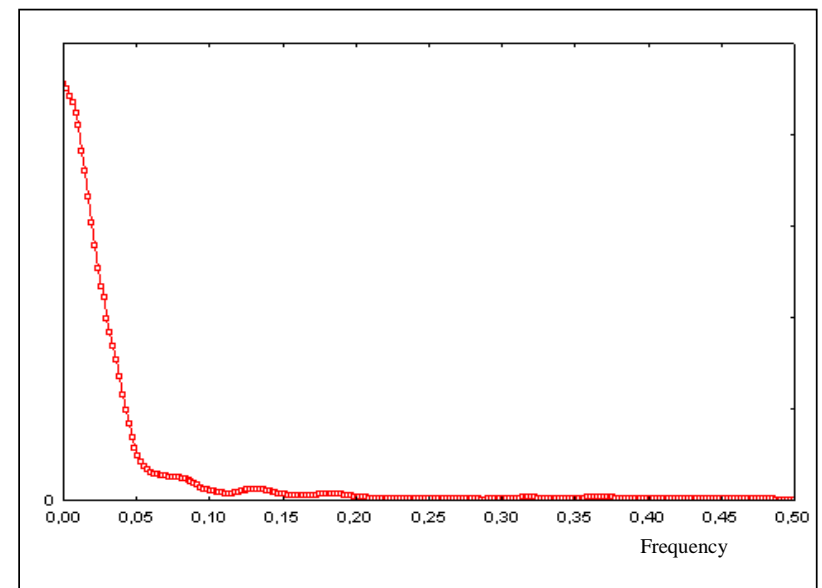

Fig. 5. Chart of spectral density functionwith approximation

Operating rotation frequency range of the crankshaft diesel engine lies between minimum stable to maximum ermissible.

Minimum stable rotating frequency speed of most diesels $73 \mathrm{rad} / \mathrm{s}(700 \mathrm{r} / \mathrm{min}) \ldots 84 \mathrm{rad} / \mathrm{s}(800 \mathrm{r} / \mathrm{min}$.) The maximum shaft rotating frequency diesel can reach are $272 \mathrm{rad} / \mathrm{s}$ (2600 r/min), but usually does not exceed 240 $\mathrm{rad} / \mathrm{s}(2290 \mathrm{rev} / \mathrm{min})$... $262 \mathrm{rad} / \mathrm{s}(2500 \mathrm{r} / \mathrm{min})$. [3] The most severe dynamic mode is the mode of acceleration of the CSD input shaft [3]. Therefore maximum perturbing action from the diesel engine on CSD will be during the transition from the bottom of the diesel engine speed to the top, corresponding to the maximum speed. This mode is characterized by the acceleration of diesel without a 
primary load; the most perturbation effect corresponds to the minimum acceleration time from the lower to the upper limit of the rotation frequency range of the shaft diesel engine, which, according to the experimental measurements, is $1.2 \mathrm{~s}$ [3]. Depending on the type of diesel engine, its design parameters, adjustments, etc., parameters of the perturbing action may change, shifting, usually, to the lower frequencies and smaller amplitudes. Diesel engine, mounted on terrestrial self-propelled machine and connected to the input link of the studied CSD has specific minimum engine speed equal to 94 $\mathrm{rad} / \mathrm{s}(900 \mathrm{r} / \mathrm{min})$, the specific maximum - $220 \mathrm{rad} / \mathrm{s}$ (2100 r/min), and the acceleration of diesel shaft less than $400 \mathrm{~min}^{-1} / \mathrm{s}$. These parameters define the limits of perturbing action on CSD from a diesel engine; therefore, in the future they can be accepted as estimated and consider only the suitable range from obtained data set. The distribution of the disturbance on the operating range of speeds for the rounded values range limits shown in Fig. 6. In this case, the required adjustment range is: $R=2,34$.

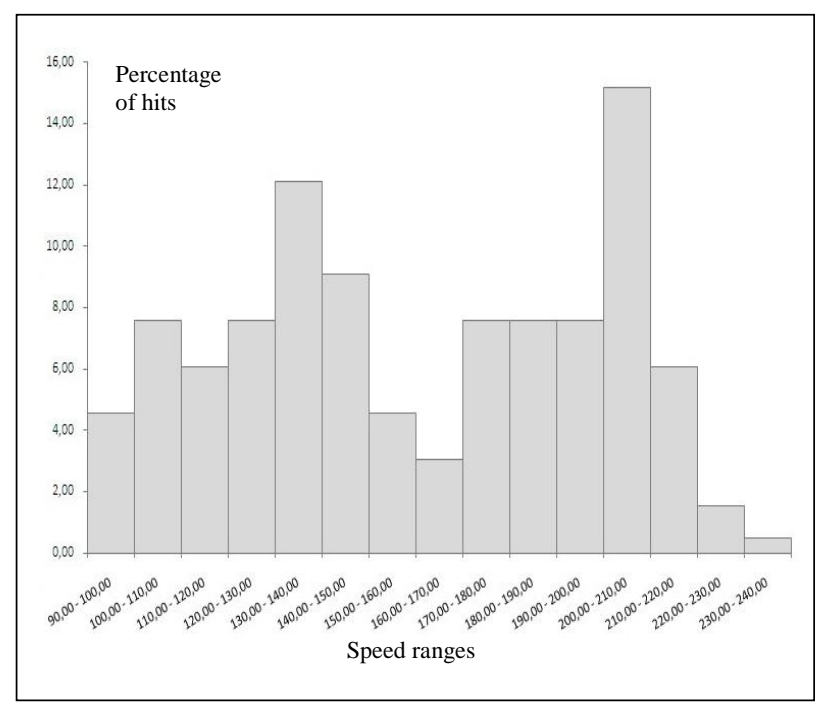

Fig. 6. The distribution of the disturbance on the operating range

In this regard the need for full-scale experiment appeared, so a research stand was developed and implemented for this purpose.

Creating of CSD (Fig. 7)stand is based on the International Scientific-Educational Center BSTUFESTO "Synergy" of the Baltic State Technical University (St.-Petersburg, Russia).

The stand consists of CSD, an electric motor combined with a volume hydraulic drive (a simulation motor with variable speed rate), and an electrical generator. A series of preliminary experiments was carried out on the stand, which aimed to define an area of zero velocities of a motor at a constant motor speed and variable load on the output shaft. As a result of a series of preliminary experiments, the data confirming the results of calculations was obtained. During the main stage of the experiments it is planned a number of tests of the drive operating in the CSD mode to identify and qualitatively assess the impact of an area of zero velocities on the accuracy of positioning of the output link.

Information, obtained as a result of ongoing researches, will serve as a basis for designing of the control system for CSD, which will take into account reversal of the motor and offset the impact area of zero velocities on the drive accuracy.

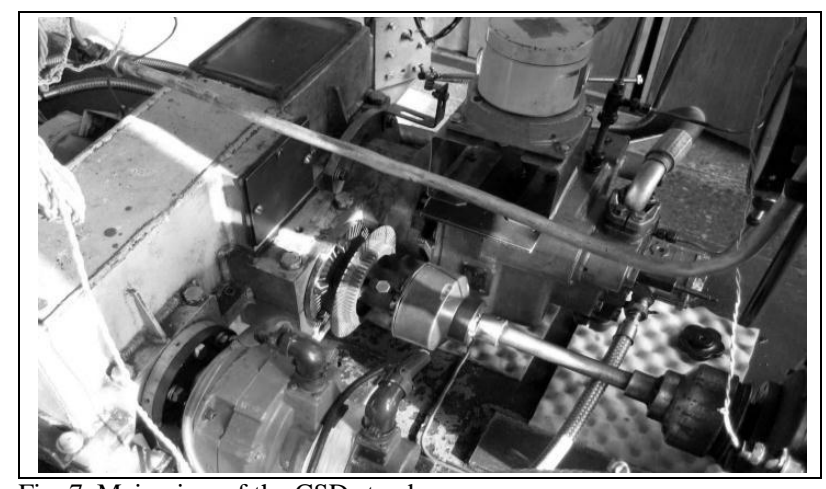

Fig. 7. Main view of the CSD stand

In addition to researches, there is the task of creating of such a methodology of design and calculation of HCSD that would allow reliably predict the accuracy of maintaining rotation speed in transition and stationary modes, weight and dimension parameters and coefficient of efficiency of the drive without the design development transmission, i.e. at the stage of choosing the structural and kinematic drive schemes.

\section{CONCLUSION}

The analysis of disturbances on CSD has practical value for a creation of a drive with the required precision characteristics, allowing studying the interconnection of the impact distribution and static characteristics of the drive and installation power of its hydraulic machines.

The interconnection, at first, allows to substantiate ways of using reserve on installed power of drive hydraulic machines and second, to identify opportunities to reduce the influence of zero velocity zone on the output characteristics of hydraulic machines [6]. One of such opportunities is to shift of the zone from the area with maximum density of the statistical distribution the input action.

\section{REFERENCES}

[1] Kanarchuk, V. (2008). Durability andengine wearunder dynamicoperating conditions, Naukova Dumka, Kiev

[2] Abdullaev, N.; Petrov U. (2005). Theory anddesign methods of optimal regulators, Energoatomizdat, St.-Petersburg

[3] Kistochkin E. (2007). Volumetrichydromechanicaltransmission. Calculation and design, Mashinostroenie, St.-Petersburg

[4] Babaev, O.M.; Ignatov, L.N.; Kistochkin, E.S. \& Cvetkov, V.A. (2000). Hydromechanical power transmissions, Mechanical engineering, St.-Petersburg, Russia

[5] Mikhaylov, M.; Larchikov, I.; Yurov, A.; Cvetkov, V. \&Stazhkov, S. (2011).Hydromechanical constant speed drive forwind-driven generators, Proceedings of the $22^{\text {nd }}$ International DAAAM Symposium, Katalinic, B. (Ed.), pp. 0235-0236, ISBN 978-3-901509-83-4, Austria Center Vienna, Vienna, Austria, October 2011, DAAAM International Vienna, Vienna

[6] Larchikov, I.; Stazhkov, S.; Mikhaylov, M.; Yurov, A. \& Cvetkov, V. (2011).Study of Hydro Mechanical Processes in Hydro Machines of Power-Intensive Drives, Proceedings of the $22^{\text {nd }}$ International DAAAM Symposium, Katalinic, B. (Ed.), pp. 0875-0876, ISBN 978-3-901509-83-4, Austria Center Vienna,Vienna, Austria, October 2011, DAAAM International Vienna, Vienna 